\title{
Polypoid Undifferentiated Carcinoma With Osteoclast-like Giant Cells Arising in the Distal Common Bile Duct: A Rare Case Report
}

\author{
XIAOYAN LIAO ${ }^{1,2}$, JANE HOULDSWORTH ${ }^{1}$, JEAN LUO ${ }^{3}$, NOAM HARPAZ ${ }^{1}$ and HONGFA ZHU ${ }^{1}$ \\ ${ }^{1}$ Department of Pathology, Mount Sinai Hospital, New York, NY, U.S.A.; \\ ${ }^{2}$ Department of Pathology, University of Rochester Medical Center, Rochester, NY, U.S.A.; \\ ${ }^{3}$ Department of Pathology, New York-Presbyterian Queens Hospital, New York, NY, U.S.A.
}

\begin{abstract}
Background: Undifferentiated carcinoma with osteoclast-like giant cells (UC-OGC) in distal common bile duct $(C B D)$ is a rare entity. Case report: This case report describes a 45-year-old male with a history of a choledochal cyst status post partial excision and cholecystectomy who presented with a mass in the remaining distal/intrapancreatic common bile duct. It was initially mistaken for post-surgery hematoma; however, the rapid growth raised concern for malignancy, and prompted a pancreaticoduodenectomy (Whipple) procedure. Macroscopic examination revealed a $5.5 \mathrm{~cm}$ polypoid mass grossly confined in the lumen of the distal CBD. Histology was consistent with UC-OGC, with minimal invasion into the polyp stalk and adjacent $C B D$ wall. Immunohistochemistry demonstrated co-expression of CK7 and p40, normal/wild-type p53, and retained SMAD4 expression in tumor cells. Next-generation sequencing detected mutations at p.Q61H (c.183A>C) of KRAS and p.E545K (c.1633G>A) of PIK3CA, keeping in line with similarity to conventional cholangiocarcinoma. The patient remained disease-free after two years of follow-up without chemotherapy. Conclusion: To our knowledge, this is the first case report of UC-OGC presented as a polypoid mass in the distal CBD. It highlights the complex dynamism and controversial pathogenesis of this unique entity, which should be made aware to avoid diagnostic pitfalls.
\end{abstract}

Extrahepatic bile duct carcinoma is rare. Primary sclerosing cholangitis and choledochal cyst are known risk factors (1). The pre-invasive neoplasms in extrahepatic biliary tract

Correspondence to: Hongfa Zhu, MD, Ph.D., Annenberg 15-38E, The Mount Sinai Medical Center, 1468 Madison Avenue, New York, NY 10029, U.S.A. Tel: +1 2122418014, e-mail: Hongfa.zhu@ mountsinai.org

Key Words: Undifferentiated carcinoma with osteoclast-like giant cells, cholangiocarcinoma, next-generation sequencing. generally present as two types: intraductal papillary neoplasms and mucinous cystic neoplasms. The invasive carcinomas are further classified into adenocarcinomas, squamous cell carcinomas, adenosquamous, and undifferentiated carcinomas (medullary type and sarcomatoid types), among other variants (2). Undifferentiated carcinoma with osteoclast-like giant cells (UC-OGC) is a unique disease entity composed of two cell types osteoclast-like giant cells and mononuclear cells (2). It has been rarely described in the pancreas $(3-7)$, gallbladder $(8,9)$ and ampullary region $(10$, $11)$, but not in the extrahepatic bile duct yet.

\section{Case Report}

The patient was a 45-year-old Asian male with a history of a choledochal cyst status post-excision and cholecystectomy. A computed tomography (CT) scan prior to cholecystectomy showed an " $8.0 \mathrm{~cm}$ large cystic structure at the head of pancreas extending to the periportal region". However, the operation was performed to the distal level of the pancreatic portion of the bile duct with intrapancreatic distal common bile duct (CBD) left in situ. Pathology report of the excised choledochal cyst and gallbladder was unremarkable. The patient developed hemorrhage 2 weeks after cholecystectomy, and returned to the operating room for management. Several months later during an interval examination, he was noted to have a mass in the intrapancreatic remaining distal CBD, which was initially considered to be residual hemorrhage/hematoma. However, the mass rapidly increased in size, measuring initially 3.9 $\mathrm{cm}$, and then $5.5 \mathrm{~cm}$ after only 2 weeks. Radiographically, the mass appeared to be well circumscribed, cystic, containing acute hemorrhage (Figure 1). There was no evidence of distant metastasis. A fine needle aspiration of the mass was performed and cytology was "positive for neoplastic cells". A pancreaticoduodenectomy (Whipple) procedure was then performed to definitively remove the mass. 
Histology and immunohistochemical studies. Macroscopic examination of the pancreas head and CBD revealed dilated $\mathrm{CBD}$ with a pedunculated polypoid mass attached to the $\mathrm{CBD}$ wall by a thin narrow stalk located approximately $1 \mathrm{~cm}$ from the ampulla. The mass measured $5.5 \mathrm{~cm}$ in greatest dimension, with variegated cut surface corresponding to areas of necrosis and hemorrhage (Figure 2A and B). Microscopically, two types of cells were observed, the numerous bland looking multinucleated giant cells resembling osteoclasts intermixed intimately with mononuclear cells that are spindly to squamoid in appearance, forming anastomosing tumor nests (Figure 3). Hemorrhage and hemosiderin-laden macrophages were present, as well as scattered small foci of tumor necrosis. Mitoses were occasionally encountered but not frequent. The invasive carcinoma was mostly confined to the polyp with minimal invasion into the stalk and adjacent CBD wall (Figure $3 \mathrm{~A}$ and $\mathrm{B}$ ). The pancreas and duodenum were not involved. $\mathrm{A}$ rare focus of invasive carcinoma protruding into a small lymphovascular space was noted (Figure 3C), but no lymph node metastasis in 15 lymph nodes submitted for examination.

Immunohistochemistry was performed on selected blocks and showed that osteoclast-like giant cells were highlighted only by CD68 (Figure 3D). The mononuclear cells were positive for CK7, CK19, EMA, with co-expression of p63, p40 and CK5/6, confirming biliary epithelial origin with squamous cell differentiation (Figure 3E). They were negative for CK20, CDX2, MUC6, and MUC2 (data not shown). Immunostaining of p53 showed scattered positivity corresponding to normal/wild-type expression pattern (Figure 3F). Mitosis index by Ki67 immunostaining was approximately $10 \%$ in tumor cells. SMAD4 expression was retained. Tumor cells were microsatellite stable as they retained expression of all four proteins including MLH1, PMS2, MSH2, and MSH6 (data not shown).

Next-generation sequencing. To better characterize this unique tumor type, next-generation sequencing (NGS) was performed on a selected block that contained $>90 \%$ tumor cells. Genomic DNA was extracted from the submitted specimen and amplified by multiplex PCR of targeted sequences in 50 genes, using the Ion AmpliSeq Cancer Hotspot Panel v2 to generate an amplicon library. The library was then clonally amplified by emulsion PCR, enriched and sequenced using the Ion AmpliSeq Cancer Hotspot Panel (v2, Thermo Fisher Scientific Inc., Waltham, USA). The detection limit of this assay is $5 \%$ mutant alleles in a background of wild-type alleles. Using this method, two mutations were identified in our case, one in KRAS at p.Q61H (c.183A >C), and the other one in PIK3CA at p.E545K (c.1633G>A). Both mutations are frequently detected in pancreatic ductal adenocarcinoma.

Follow-up. The patient was discharged from hospital without any complications. He refused chemotherapy. Two years after surgery, he remains disease-free, with follow-up abdominal CT scan showing no evidence of tumor recurrence.

\section{Discussion}

UC-OGC was initially reported in the pancreas by Juan Rosai in 1968 (12), and the World Health Organization in 2000 defined it as a new variant of pancreatic ductal adenocarcinoma (PDAC), accounting for less than $1 \%$ of the pancreatic tumors (2). Carcinoma with OGC was subsequently described in the hepatobiliary tree, including the liver (13) and gallbladder (14). However, due to its rarity and lack of unified diagnostic terminology, the clinicopathological features of this entity in the biliary tree are unclear. As a result, WHO and recent AJCC guidelines did not include UC-OGC as a distinct disease entity in the biliary system. We thus present the very first UC-OGC as a variant of cholangiocarcinoma occurring in the extrahepatic bile duct, that was confirmed by immunohistochemistry, showing ductal differentiation and by molecular studies, bearing similarity to conventional cholangiocarcinoma.

In this unique case, the predominant polypoid configurations with minimal stalk invasion raise a possibility that this entity can be somewhere pre-invasive. As a matter of fact, the cytology from the original fine needle aspiration on this mass was initially interpreted as "low-grade atypia" by outside pathologists, a pitfall that exaggerated the importance of keeping awareness of this entity for reaching appropriate diagnosis. Despite the polypoid growth configuration and deceivingly low-grade cytology, KRAS and PI3KCA mutations were both detected in the tumor cells. Since $K R A S$ and $P I K 3 C A$ genes are frequently co-mutated in cholangiocarcinoma patients (15), detection of both mutations reveals its similarity to conventional cholangiocarcinoma and aggressiveness in nature. Indeed, the rapid growth of this lesion on radiology, and the rare focus of lymphovascular space invasion despite negative lymph nodes, indicate its malignancy in nature.

Although we believe this is the first case report of UCOGC in extrahepatic bile duct, a few similar entities in the literature were reported and deserve review. Giant cell tumor (GCT) of the extrahepatic biliary tree, which bears its name and histology analogy to the bone counterpart (16-18), is considered benign, although it is also composed of a mixture of mononuclear and multinucleated osteoclast-like giant cells. However, different than UC-OGC, the mononuclear cells in GCT do not express any cytokeratin, indicating mesenchymal rather than epithelial origin, as seen in our case. On the other hand, rare case reports of giant cell tumorlike cholangiocarcinoma of hepatic hilus (19), and sarcomatoid cholangiocarcinoma with osteoclast-like giant cells in the CBD (20) were reported, which may bear intrinsic similarity to our case, where the mononuclear cells 


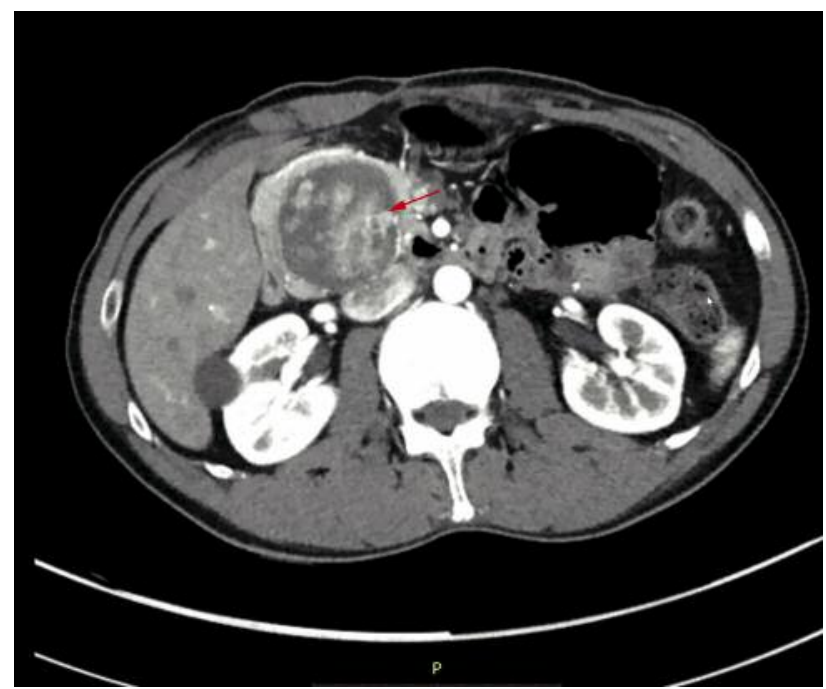

Figure 1. Radiological imaging revealing a large mass (red arrow) in the pancreatic head.

were frequently positive for CK7 and CK19. However, the mononuclear cells in those cases demonstrated high-grade cytology and frequent mitosis that are readily distinguishable from those in our case or in benign GCT. Nevertheless, given the confusing terminology used in literature, we advocate using UC-OGC, the same as in the pancreas counterpart, whenever the bile duct epithelial origin and malignant nature can be confirmed by immunostaining and/or molecular studies. This unified term can also facilitate future systemic reviews to better characterize its clinicopathological features.

The influence of OGC on the patient is controversial. In the breast, although some suggested that the presence of OGC indicates a less aggressive tumor with a good outcome (21), in approximately one third of cancers with osteoclast-like giant cells there is axillary node metastasis, leading to a worse prognosis (22). In pancreas, the prognosis of UC-OGC is considered poor (6), but still better than conventional pancreatic ductal adenocarcinoma (PDAC) or UC-OGC with associated PDAC (23). Besides the presence of OGC, older age, male gender, tumor size, node and distant metastases, and a concomitant component of ductal adenocarcinoma are probably more influential factors on prognosis during the clinical course.

One clinical feature worth mentioning is that patients with pancreatic and ampullary UC-OGC are frequently asymptomatic so that tumors may not be detected until they are quite large and have reached advanced stages with widespread metastases (13). In our case, the patients presented no symptoms, likely also due to the intraductal/intracystic growth pattern. In such situations, close follow-up in patients with a mass lesion in the abdomen, keeping high suspicion on rapid growth, and accurate pathology assessment on the nature
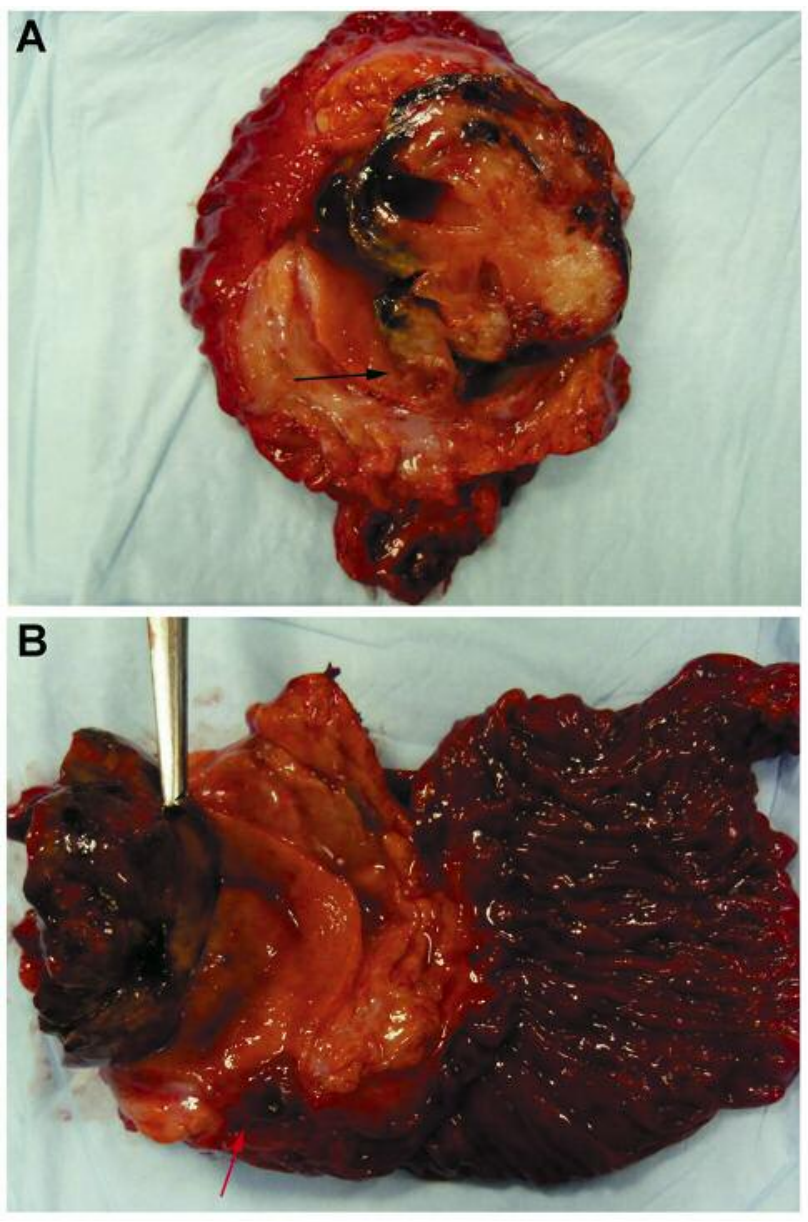

Figure 2. Gross examination of the mass. The tumor is located in the distal $C B D$, with a think stalk attached (black arrow) to the CBD wall (A). It is located approximately $1 \mathrm{~cm}$ from the ampullary region (red arrow) (B).

of this lesion are critical for proper management. For resectable tumors in the pancreas, ampullary region, and distal CBD, pancreaticoduodenectomy seems to be the treatment of choice, and a future large case series with longterm follow-up are necessary to clarify the surgical outcome and effects of chemotherapy in patients with UC-OGCs at different anatomic sites.

In summary, we report the first case of UC-OGC in distal $\mathrm{CBD}$ as a pedunculated polypoid mass with minimal invasion into adjacent tissue. Immunohistochemistry and molecular studies confirmed the cholangiocarcinoma nature of this entity. We propose to use UC-OGC, the same terminology described in the pancreas, as a unifying term to avoid confusion, as well as to facilitate future follow-up studies. We believe that the patient's history of choledocyst was likely a predisposing factor for developing UC-OGC, although the 

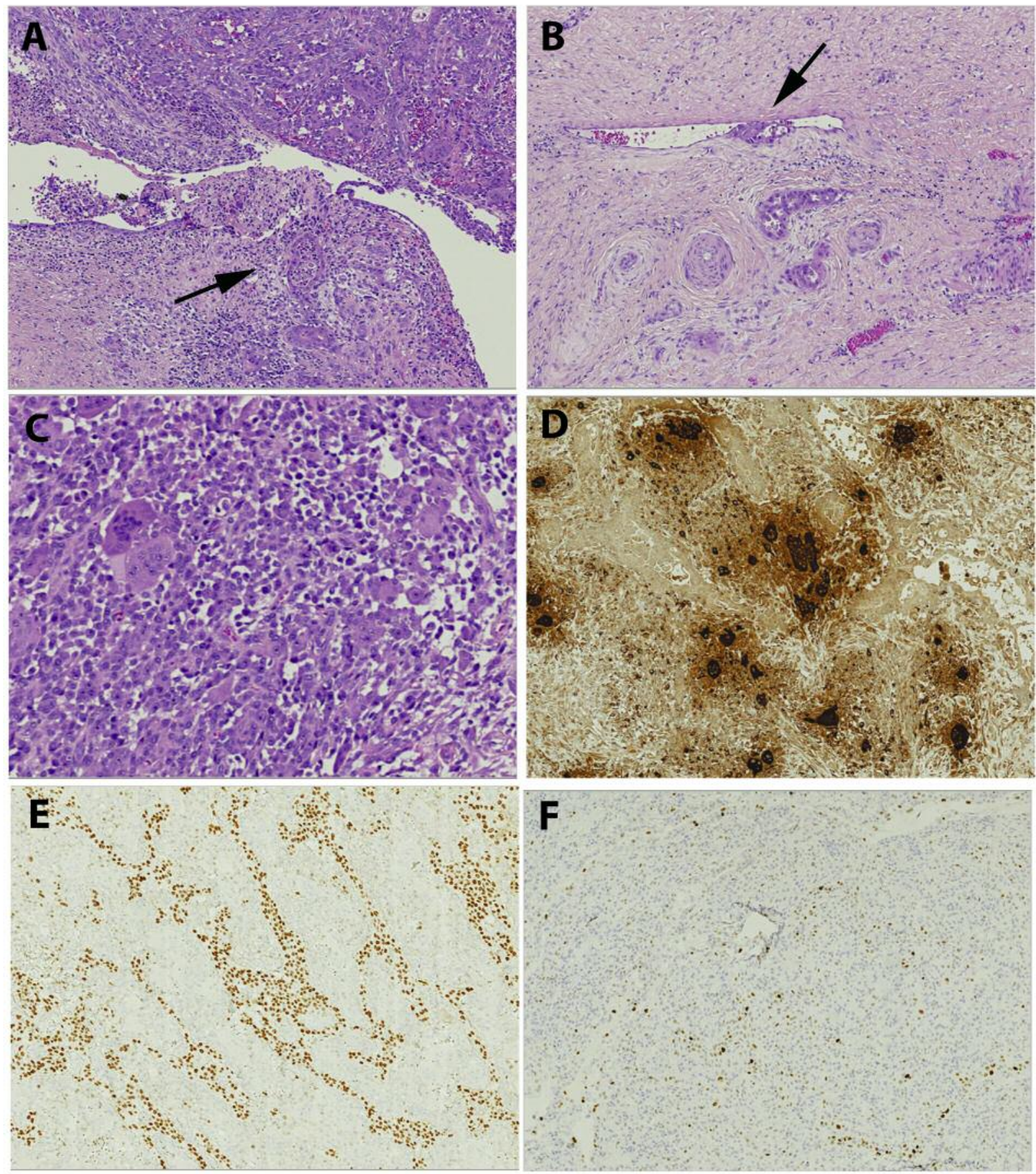

Figure 3. Histological features of the intraductal UC-OGC, showing minimal stalk invasion (A, arrow), with a small focus of lymphovascular space invasion identified $(B$, arrow). The tumor cells are composed of two types $(C)$. The osteoclast-like giant cells are positive for CD68 $(D)$, while the mononuclear cells are positive for p63 (E). p53 shows wild-type expression (F). Magnification: A, B, D, E, F: 200x, C: 400x.

exact pathogenesis is still unclear. Similar to UC-OGC in pancreas, as a variant of PDAC, UC-OGC in biliary system likely represents a variant of cholangiocarcinoma, and may bear favorable prognosis if detected early and removed completely by surgery.

\section{References}

1 Odze RD and Goldblum JR: Odze and Goldblum surgical pathology of the GI tract, liver, biliary tract, and pancreas. Elsevier Canada, 2014. 
2 Bosman FT. Carneiro F, Hruban RH and Theise ND: WHO classification of tumours of the digestive system. Lyon: International Agency for Research on Cancer, 2010.

3 Maksymov V, Khalifa MA, Bussey A, Carter B and Hogan M: Undifferentiated (anaplastic) carcinoma of the pancreas with osteoclast-like giant cells showing various degree of pancreas duct involvement. A case report and literature review. JOP 12 : 170-176, 2011.

4 Wada T, Itano O, Oshima G, Chiba N, Ishikawa H, Koyama Y, Du W and Kitagawa Y: A male case of an undifferentiated carcinoma with osteoclast-like giant cells originating in an indeterminate mucin-producing cystic neoplasm of the pancreas. A case report and review of the literature. World J Surg Oncol 9: 100, 2011.

5 Jo S: Huge undifferentiated carcinoma of the pancreas with osteoclast-like giant cells. World J Gastroenterol 20: 2725-2730, 2014.

6 Kobayashi S, Nakano H, Ooike N, Oohashi M, Koizumi S and Otsubo T: Long-term survivor of a resected undifferentiated pancreatic carcinoma with osteoclast-like giant cells who underwent a second curative resection: A case report and review of the literature. Oncol Lett 8: 1499-1504, 2014.

7 Gao HQ, Yang YM, Zhuang Y and Liu P: Locally advanced undifferentiated carcinoma with osteoclast-like giant cells of the pancreas. World J Gastroenterol 21: 694-698, 2015.

8 Manouras A, Genetzakis M, Lagoudianakis EE, Markogiannakis H, Papadima A, Agrogiannis G, Gakiopoulou H, Kekis P, Filis $\mathrm{K}$ and Patsouris E: Undifferentiated giant cell type carcinoma of the gallbladder with sarcomatoid dedifferentiation: a case report and review of the literature. J Med Case Rep 3: 6496, 2009.

9 Akatsu T, Kameyama K, Kawachi S, Tanabe M, Aiura K, Wakabayashi G, Ueda M, Shimazu M and Kitajima M: Gallbladder carcinoma with osteoclast-like giant cells. J Gastroenterol 41: 83-87, 2006.

10 Matsuzawa G, Shirabe K, Gion T, Tsujita E, Ooya M, Kajiyama $\mathrm{K}$ and Nagaie T: Surgically resected undifferentiated carcinoma with osteoclast-like giant cells of the periampullary region involving the orifice of the papilla of Vater: Report of a case. Surg Today 40: 376-379, 2010.

11 Kawamoto Y, Ome Y, Terada K, Hashida K, Kawamoto K and Ito $\mathrm{T}$ : Undifferentiated carcinoma with osteoclast-like giant cells of the ampullary region: Short term survival after pancreaticoduodenectomy. Int J Surg Case Rep 24: 199-202, 2016.

12 Rosai J: Carcinoma of pancreas simulating giant cell tumor of bone. Electron-microscopic evidence of its acinar cell origin. Cancer 22: 333-344, 1968

13 Sasaki A, Yokoyama S, Nakayama I, Nakashima K, Kim YI and Kitano S: Sarcomatoid hepatocellular carcinoma with osteoclastlike giant cells: case report and immunohistochemical observations. Pathol Int 47: 318-324, 1997.
14 Njoumi N, Elalami FH, Attolou G, Saoud O, Elabsi M, Echarrab M, Elouannani M, Errougani A, Amraoui M and Chkoff MR: Undifferentiated pancreatic carcinoma with osteoclast-like giant cells: a case report. J Gastrointest Cancer 45: 96-98, 2014.

$15 \mathrm{Xu}$ RF, Sun JP, Zhang SR, Zhu GS, Li LB, Liao YL, Xie JM and Liao WJ: KRAS and PIK3CA but not BRAF genes are frequently mutated in Chinese cholangiocarcinoma patients. Biomed Pharmacother 65: 22-26, 2011.

16 Albores-Saavedra J, Grider DJ, Wu J, Henson DE and Goodman ZD: Giant cell tumor of the extrahepatic biliary tree: a clinicopathologic study of 4 cases and comparison with anaplastic spindle and giant cell carcinoma with osteoclast-like giant cells. Am J Surg Pathol 30: 495-500, 2006.

17 Wang DD, Zheng YM, Teng LH, Sun YN, Gao W, Wang LM, Wang YH, Li F and Lu DH: Benign giant-cell tumor of the common bile duct: a case report. World J Gastroenterol 20: 15448-15453, 2014.

18 Kolokotronis T, Glanemann M, Wagner M, Bohle RM and Grünhage F: Giant cell tumor of the distal common bile duct: report of a rare, benign entity that may mimic malignant biliary obstruction. Int J Colorectal Dis 29: 413-414, 2014.

19 Haratake J, Yamada H, Horie A and Inokuma T: Giant cell tumor-like cholangiocarcinoma associated with systemic cholelithiasis. Cancer 69: 2444-2448, 1992.

20 Kim HM, Kim H and Park YN: Sarcomatoid cholangiocarcinoma with osteoclast-like giant cells associated with hepatolithiasis: A case report. Clin Mol Hepatol 21: 309-313, 2015.

21 Perez-Alonso P: Breast carcinoma with osteoclast-like giant cells diagnosed by fine-needle aspiration cytology. Diagn Cytopathol 40: 148-149, 2012.

22 Cai G, Simsir A and Cangiarella J: Invasive mammary carcinoma with osteoclast-like giant cells diagnosed by fineneedle aspiration biopsy: review of the cytologic literature and distinction from other mammary lesions containing giant cells. Diagn Cytopathol 30: 396-400, 2004.

23 Luchini C, Pea A, Lionheart G, Mafficini A, Nottegar A, Veronese N, Chianchiano P, Brosens LA, Noë M, Offerhaus GJA, Yonescu R, Ning Y, Malleo G, Riva G, Piccoli P, Cataldo I, Capelli P, Zamboni G, Scarpa A and Wood LD: Pancreatic undifferentiated carcinoma with osteoclast-like giant cells is genetically similar to, but clinically distinct from, conventional ductal adenocarcinoma. J Pathol 243: 148-154, 2017.

Received November 28, 2018

Revised December 6, 2018

Accepted December 7, 2018 